\title{
Las equivalencias formales de la actualización nominal en castellano y quechua
}

\author{
Norma I. Meneses T. \\ Departamento Académico de Lingüística
}

Este artículo, cuyo tema es el contacto entre el castellano y el quechua, constituye la presentación de los resultados de un análisis contrastivo entre el castellano estándar y el quechua ayacuchano, en relación a las formas de manifestación de la actualización nominal en ambas lenguas.'

El objetivo del estudio fue establecer los morfos de manifestación de la actualización nominal en castellano y en quechua, y cómo estas formas de manifestación, salvando las distancias propias del uso idiomático en cada lengua, pueden funcionar como equivalentes formales (EF) entre sí.

La premisa fundamental que nos guía es la consideración que la traducción y la interferencia constituyen dos caras de un mismo proceso de equiparación lingüística. En efpthner:easê, Genemês una tequiparación eficiente; en el segundo, una equiparadrongungüstich deficienteverso "

Desde esta perspectiva, las hipótesis que nos planteamos fueron: a) la función actualizadora es de carácter universal y pertenece al plano del contenido en cada lengua, constituyendo el punto de encuentro funcional entre ellas; b)esta función se manifiesta mediante formas distintas en el plano de la expresión de cada lengua, evidenciando los puntos de desencuentro gramatical; c) estos elementos de desencuentro gramatical constituyen equivalentes formales entre sí en ambas lenguas.

Este análisis contrastivo fue el tema de mi tesis de licenciatura. La acttralización nominal en el castellano y el quechua EAP de Linguiistica, Facultad de Letras, UNMSM. 1995.

Letras (Lima), 95-96: 171-186, 1998. 


\section{Antecedentes}

La revisión de antecedentes trajo como resultado la comprobación de la falta de estudios contrastivos entre el castellano y las diversas lenguas amerindias. Salvo un estudio contrastivo de índole fonológico entre el castellano y el quechua de la Dra. Madelaine Zúñiga no existía ninguno referido al contraste gramatical.

La búsqueda de una adecuada metodología para emprender esta tarea, hizo necesaria la revisión de un numeroso material teórico proveniente de las diversas corrientes conceptuales de la lingüística teórica y aplicada. Finalmente, tomamos como principales fuentes los planteamientos de J. Catford, E. Coseriu y $\mathrm{U}$. Weinreich. El primero, un reconocido lingüista inglés de orientación funcionalista y los dos últimos, renombrados lingüistas estructuralistas. ${ }^{2}$ En realidad, se trató de conjugar diversos conceptos propios y ajenos, nuevos y antiguos, con la exclusiva finalidad de esbozar una metodología de análisis contrastivo que de manera homıgénea fuera posible aplicar a la descripción de dos lenguas tipológicamente tan diferenciadas como son el castellano y el quechua.

\section{Marco teórico}

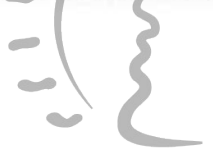

\section{Biblioteca de Letras}

El marco teórico que sustenta lametodologíade este análisis contrastivo es uno de carácter funcional; pero desde una perspectiva universalista de la concepción del lenguaje. Es decir, que concebimos que existe una relación dialéctica de lo universal frente a lo particular entre el lenguaje humano y cada lengua natural. La capacidad humana de hablar se organiza en torno a una sistematización universal de categorías funcionales las cuales adquieren una forma específica -dentro de un estrato gramatical ${ }^{3}$ y un nivel de estructuración lingüística definidos ${ }^{4}$ - propia de una lengua natural dada.

: Aunque directamente no tomamos los planteamientos de N. Chomsky, para la elaboración de nuestra metodología de análisis, sí le debemos nuestra concepción del carácter universal del lenguaje. El enfoque funcionalista señala que los estratos gramaticales son el morfema, la palabra, la frase, la propo-
sición. la oración y el texto.

4 Nos referimos a los componentes de la estructura de una lengua: fonología, morfología, sintaxis y léxico. Por ejemplo, la estructuración lingüística de la interrogación total directa ( preguntas que requieren una respuesta sí o no) se produce en el componente morfológico, en quechua; en el componente fonológico, en castellano y en el componente sintáctico, en el inglés. 
Basándonos en lo anterior, el método empleado es uno de tipo hipotéticodeductivo y de orientación onomasiológica, en un primer momento, con la finalidad de establecer las formas de estructuración paradigmática de la función actualizadora en ambas lenguas (cuadros 1 y 2). Es decir, que en esta primera etapa se obtienen las formas de manifestación de la actualización nominal para cada lengua. En una segunda etapa, el método adquiere una carácter más inductivo pues el objetivo es la descripción particular de los usos gramaticales de las formas de la actualización nominal en cada lengua. Para esta etapa empleamos corpus provenientes de descripciones gramaticales, de literatura y de informantes.

Sobre la base de estas descripciones homogéneas y como resultado del análisis contrastivo se produce el establecimiento de las equivalencias formales entre ambas lenguas. Es decir, que el análisis contrastivo, nos ha de dar no sólo las diferencias entre ambas lenguas; sino también los elementos análogos, por su función, en ambas lenguas. Estos puntos de encuentro funcional entre ambas lenguas constituyen los equivalentes formales para los cuales se deben establecer clara y respectivamente sus limitaciones sintagmáticas en cada lengua.

Por lo tanto, por esta forma de obtención, los EF difieren grandemente de las correspondencias formales y de las equivalencias de traducción establecidas por J.C. Catford. Como se sabe, las correspondencias formales se establecen como un simple inventario a la vista de elementos gramaticales, que perteneciendo al mismo estrate gramatical en Gubas tenguas, cumplen una función análoga al interior dek sistemma en cada ahả deellası'Bor ejemplo: el pronombre de 1ra. persona singular en función sujeto es 'yo' en castellano, 'I' en inglés. Este procedimiento resulta poco productivo dado la capacidad creativa de cada lengua para crear formas de manifestación y en todo caso, aplicable a lenguas que tengan algún acercamiento tipológico.

En cambio, los equivalentes de traducción constituyen elementos gramaticales con funciones análogas en cada lengua, que pueden pertenecer a estratos diferentes dentro del sistema integral de cada una; pero que a diferencia de los equivalentes formales son obtenidos por un procedimiento empírico-inductivo. Este se basaría en la recolección de la mayor cantidad posible de corpus, obtenidos de bilingües totalmente competentes, para poder llegar a obtener, con auxilio estadístico, las equivalencias de traducción entre ambas códigos y sus respectivos contextos (lingüísticos y extralingüísticos) de traductibilidad. Indudablemente, estos equivalentes de traducción tendrían un alto valor de eficiencia, pues darían cuenta de los contextos discursivos; pero implicaría una labor monumental y hasta cierto punto incompleta, pues no habría límites precisos 
entre los hechos propios de la estructuración del código mismo y la actividad del uso discursivo del código. Con respecto a los equivalentes formales, nuestro objetivo fundamental, fue encontrar la manera de obtener los elementos estructurales en cada lengua que cumplen la misma función, perteneciendo o no al mismo estrato gramatical.

El planteamiento ulterior acerca de estos equivalentes formales es que pueden constituir elementos fácilmente programables como formas básicas para una etapa inicial de la traducción entre ambas lenguas. Obviamente, la traducción final tendría necesariamente que incorporar aspectos relativos a las técnicas discursivas de cada lengua.

\section{Definiciones básicas}

La actualización. Según E. Coseriu, es una operación determinativa fundamental y primaria, por la cual los nombres que significan seres u objetos «virtuales» pertenecientes al conjunto total de la significación existente en una lengua se «actualizan»(concretizan) mediante la técnica del hablar (gramática) de esa lengua particular. Los elementos que cumplen esta operación se denominan actualizadores. Algunas lenguas presentan un esquema formal (morfema) que representa a esta función; pero en otras es representada de manera secundaria e implícita por esquernis fornales ceyas funcientes peincipales son otras. En castellano, esta función se expresa eqnel articulpe Este, concepto, correcto en esencia, nos parecía incompleto y consideramos, desde una perspectiva funcional, que esta función no es sólo una operación de salida - que de hecho lo es en el plano de la expresión de las gramáticas particulares - sino como una categoría funcional del nivel general de la denotación que refleja un valor designativo universal al expresar la orientación temporal de el ente conocido, el ente no conocido y el ente en sí mismo. Es decir, reconociéndole un valor deíctico de definición $n^{5}$. Por lo tanto, asumimos que las posibilidades de denotación de esta función son tres:

Stockwell et. al. reconocen el carácter deictico del artículo y establecen hasta tres clases de artículo para el inglés: el definido, indefinido y universal. Stockwell et al, The gramatical structures of English and Spamsh. University of Chicago Press. Chicago 1965, p. 65.

Lyons también lo considera como tal: “Una nueva categoría interdependiente con el caso en muchas lenguas la constituye la categoría déctica de la definición (que en español viene señalada por el artículo el, la, los. las)m, Intraduccioin a la lingziística tecirica. Ed. Teide, Barcelona, 1971, p. 307.

Dentro de la pragmática, algunos reconocen esta orientación temporal en el estrato del discurso. especialmente en la relación de interenunciados. 
Actualización definida. La existencia particular del objeto denotado es presupuesto por el hablante como de conocimiento general. Es decir, el elemento denotado es conocido por el hablante y éste presupone que también lo es por el oyente. Si es así, se debe a que el elemento denotado ha sido previamente mencionado en la comunicación. v.g. 'el hombre vino a buscarte' (el que conocemos); o simplemente el elemento denotado por su carácter único dentro del entorno (contexto) del discurso es conocido por ambos, v.g. 'El presidente'(del club). Tradicionalmente esta función ha sido reconocida como propia de la partícula gramatical denominada artículo definido. ${ }^{6}$

Actualización indefinida. La existencia particular del objeto es sólo de conocimiento particular del hablante, pero no así del oyente. El elemento denotado es uno relativamente conocido por el hablante y del que éste sólo sabe (o desea hacer saber) su número y su pertenencia a una clase de objetos. v.g. «Un hombre vino a buscarte». "Unos alumnes vinieron a saludarlo». La gramática tradicional reconoce esta función bajo la forma del artículo indefinido.?

Actualización universal. La existencia universabdel elemento denotado, es de conocimiento universal. Desde la perspectiva del hablante, el elemento denotado es uno conocido para él, para el oyente y para todos las demás personas de la comunidad integral.

Este conocimientouniversal usualmente se realiza cuando se hace referencia a la existencia del concepto en si musmo Múchas lenguas, como el castellano, no presentan un morfemalespectaltzad8parar'ssta” función, v.g. 'El hombre es bueno en esencia'. En otras, es señalado por el morfema cero como en el

Bello señala con toda claridad el valor orientativo de este actualizador, con respecto a las dos personas del discurso. Caracteriza al artículo definido como un adjetivo con cierto valor demostrativo. Bello. Andrés y J Cuervo. Grameitica de la lengua castellana, ed. revisada por R.J. Cuervo, Ed. A Roger y F. Chernoviz. Paris. 1891.pp. 67-68.

Sin embargo. numerosos lingtiistas cuestionan esta aserción y consideran que se trata mas bien de adjetivo indefinido. En rualidad. la cuestión esta aún irresuelta. Personalmente nos adscribimos a la posición tradicional porque si bien es innegable el valor como adjetivo indefinido en enunciados plurales como umos ninins vinierm: no es menos cierto que su valor es indudablemente de adjetivo numeral en traje tun lihm tury.

* En gran medida incorporamos aquí. la concepción husserliana de la significación del lenguaje. Esta concepción comprende tres formas de significación de un signo linguístico: el significado que tiene en mente el hablante del elemento referido, el elemento referido en sí mismo y el concepto o abstracción de esta representación perteneciente al código. Esto último explica el valor universal de este actualizador, pues al pertenecer directamente al código resulta de conocimiento general de la comunidad lingǘstica. 
inglés, v.g. '0 man is essentially good' ${ }^{9}$ La gramática tradicional no hace mención específica de esta actualización. ${ }^{10}$

Limitación sintagmática. Término propuesto por U. Weinreich " , que se refiere al contexto sintáctico obligatorio para la ocurrencia de un elemento estructural cualquiera. Por ejemplo, el pronombre 'yo' del castellano tiene como limitación sintagmática su empleo como Sujeto.

Morfema \{nil\}. Tomamos este término de J.C. Catford ${ }^{12}$, para referirnos a los morfemas que existen en tanto función pero no poseen un morfo propio de manifestación. Esta manifestación puede hallarse en otro estrato gramatical; o pertenecer, dentro del mismo estrato, a otra función. Es decir que un morfema \{nil\} manifiesta la función bajo morfos que cumplen simultáneamente otra función de mayor envergadura o que neutralizan su función principal dentro de un contexto sintáctico específico (limitación sintagmática). Por ejemplo, en castellano, no existe un morfo propio para actualizar un concepto universal y para ello se emplea el morfo del artículo definido: 'El hombre es bueno por naturaleza'.

Los conceptos definidos nos han permitido establecer que estas tres funciones actualizadoras se encuentran presentes en el castellano y el quechua. Sin embargo, sus formas de manifestación en el nivel particular de la gramática difieren en gran medidądebido a la diferencia tipológica de ambas lenguas.

En el castellano dbsegeamos quie đal manifestacion de la función de actualización nominal se da a través de partículas independientes conocidas como artículos. Conforman un pequeño sistema paradigmático incompleto porque no presenta una forma propia para la expresión de la función actualizadora universal, por lo que asumimos el concepto de un morfema NIL: existe la función pero no presenta una morfo propio. Para la manifestación de este último, el

\footnotetext{
". El enfoque pragmático, scñala que este actualizador tiene valor no sólo a nivel del enunciado nominal sino a nivel de todo el enunciado.

in Las gramáticas tradicionales parten del estudio de las formas gramaticales de expresión del contenido, y al parecer este actualizador no presenta una forma especializada en ninguna de las lenguas estudiadas por estas gramáticas. Stockwell en su estudio contrastivo del inglés-castellano $(O p$. cit. ) lo discrimina y le asigna una forma cero para el inglés. Amado Alonso le reconoce este mismo valor al artículo definido en algunos contextos estilísticos y en otros le asigna una forma cero. Para Alonso, esta diferenciación es esencialmente un fenómeno estilístico.

11 U. Weinreich. Lenguas en contacio, 1964, pp. 71-76.

12 J.C. Catford. A lingmistic theory of translation, pp. 27-34, Oxford University Press. London, 1967.
} 
sistema del castellano emplea los morfos propios de la actualización definida, indefinida y el morfo cero.

En el caso de los dos primeros sólo el contexto lingüístico y/o extralingüístico nos permite advertir la función de actualizador universal que cumplen en ese momento. El cuadro $\mathrm{N}^{\circ} 1$ que a continuación presentamos explicita la estructuración paradigmática de la actualización nominal en castellano. Es decir, cómo son sus formas de manifestación.

\section{Cuadro $\mathrm{N}^{\circ} 1$ \\ LA ACTUALIZACIÓN NOMINAL EN CASTELLANO ESTRUCTURACIÓN PARADIGMATICA DE LA FUNCIÓN ACTUALIZADORA}

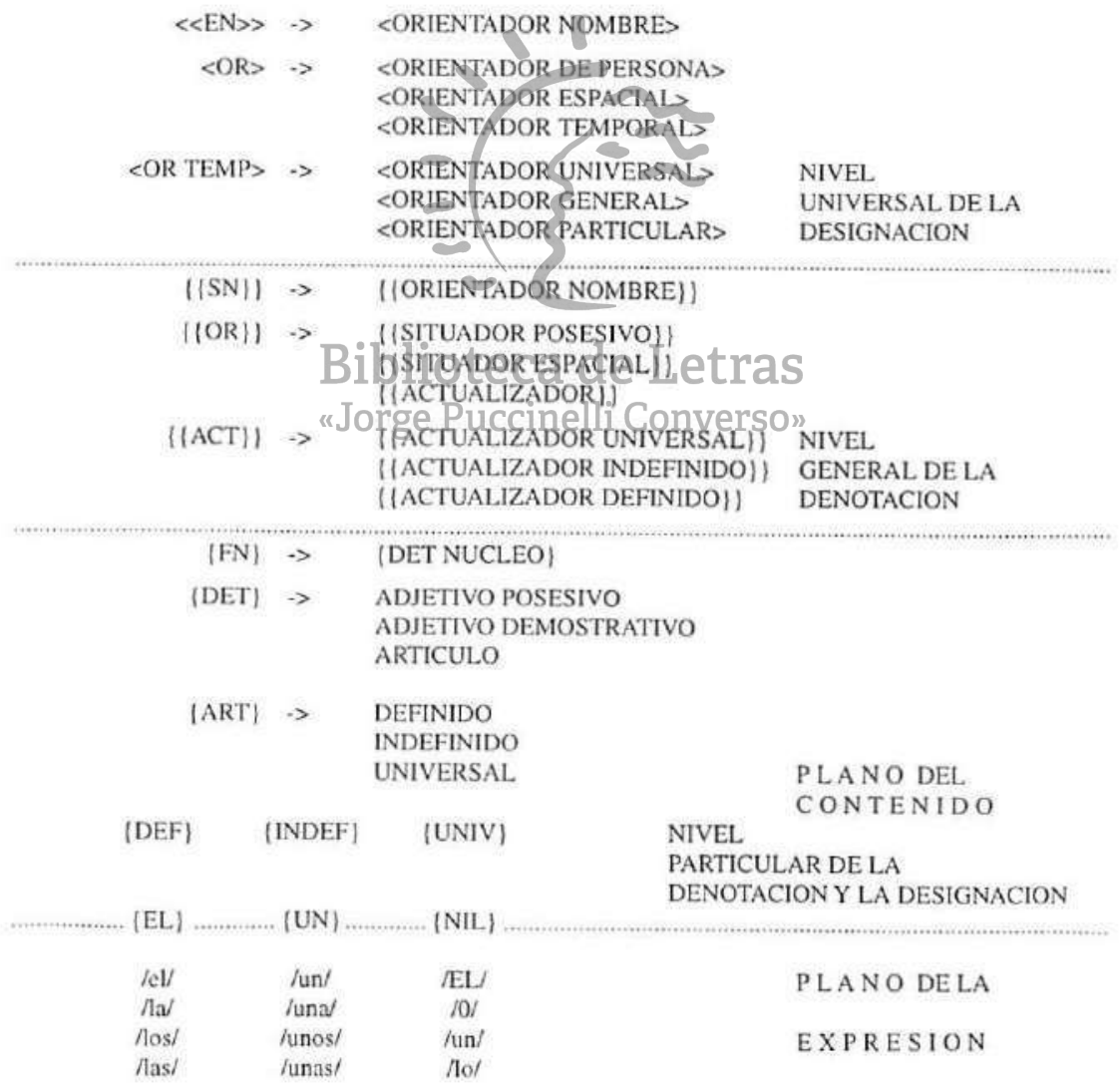


En el quechua, la actualización nominal no posee una manifestación morfológica simétrica a su estructuración paradigmática funcional. No sólo no posee partículas libres sino que no tiene morfos especializados para los actualizadores nominales. Sin embargo, hemos podido observar cierta estabilidad en el empleo de la partícula huk y el morfo cero para los actualizadores indefinido y universal, respectivamente, que podría asumirse que constituyen una suerte de artículos.

\section{Cuadro $\mathrm{N}^{\circ} 2$ \\ LA ACTUALIZACION NOMINAL EN QUECHUA ESTRUCTURACION PARADIGMATICA FUNCIONAL}

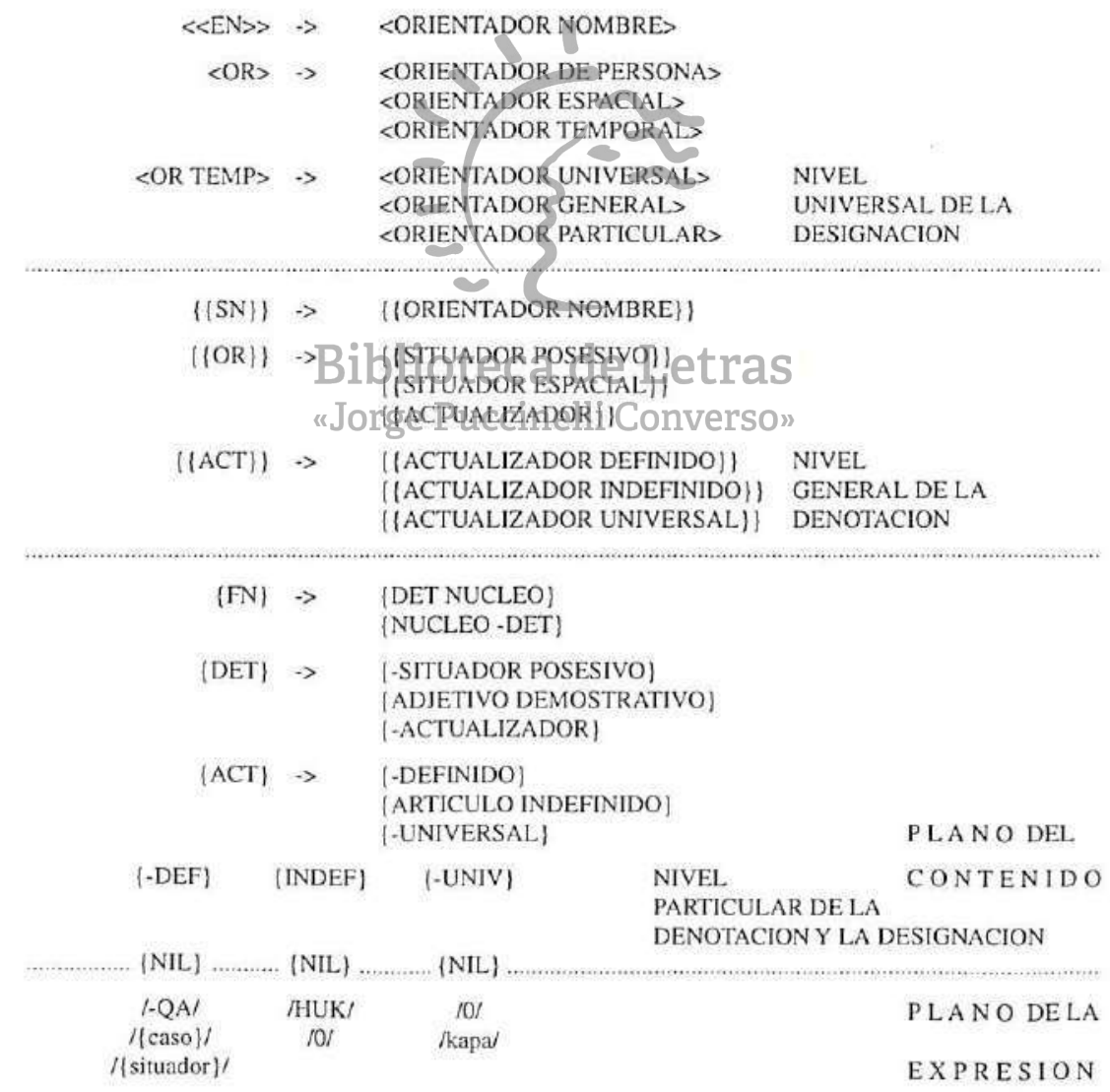




\section{COMPARACION DE LA ESTRUCTURACION MORFOLOGICA ENTRE EL CASTELLANO Y EL QUECHUA}

El cuadro $\mathrm{N}^{\circ} 3$ muestra el sistema de oposiciones entre Los tres actualizadores nominales en castellano. Se puede observar que el actualizador universal puede manifestarse en la frase nominal mediante el empleo de los morfos del actualizador definido, el actualizador indefinido y el morfo cero, tanto en la función sintáctica de sujeto como de complemento. Obsérvese las siguientes oraciones:

Función de sujeto:

El niño es bueno por naturaleza.

Un niño es bueno por naturaleza.

ONiñez y Oancianidad, dos extremos de la vida humana.

Función de complemento:

No se debe abandonar a un niño enfermo

No se debe abandonar al niño enfermo.

El verdadero misionero abandona Ofamilia y Otrabajo.

\section{Biblioteradrodie Letras}

CONFIGURACIONMORFOLOGIGA DE LA ACTUALIZACIÓN EN

CASTELLANO EN LA FN EN FUNCIÓN SINTÁCHICA DE SUJETO:

\begin{tabular}{|c|c|c|c|c|c|}
\hline \multirow[b]{2}{*}{ ACT. DEFINIDO $\{\mathrm{e}\}\}$ : } & \multicolumn{2}{|c|}{ SINGULAR } & \multirow[b]{2}{*}{ neutro } & \multicolumn{2}{|c|}{ PLURAL } \\
\hline & $\begin{array}{c}\text { MASC } \\
\text { tel }\end{array}$ & $\begin{array}{l}\text { FEM. } \\
/ \mathrm{la} /\end{array}$ & & $\begin{array}{c}\text { MASC, } \\
\text { nos/ }\end{array}$ & $\begin{array}{c}\text { FEM. } \\
\text { /las }\end{array}$ \\
\hline ACT. INDEFINIDO (un) : & lun/ & funa/ & & /unos' & iunas! \\
\hline ACT. UNIVERSAL \{nil\}: & $\begin{array}{l}\text { lel/ } \\
\text { /un/ } \\
\text { /0/ }\end{array}$ & $\begin{array}{c}\mathrm{a} / \\
\text { /una/ } \\
10 /\end{array}$ & hol & Mos/ & Ilas! \\
\hline
\end{tabular}

EN LA FN EN FUNCIÓN SINTÁCTICA DE COMPLEMENTO

\begin{tabular}{|c|c|c|c|c|c|}
\hline & INGULA & PLUR & & & \\
\hline ACT. DEFINIDO $\{\mathrm{el}\}$ : & $\begin{array}{c}\text { MASC. } \\
\text { lel/ }\end{array}$ & $\begin{array}{c}\text { FEM. } \\
\mathrm{Ala} /\end{array}$ & neutro & $\begin{array}{c}\text { MASC. } \\
\text { hlos/ }\end{array}$ & $\begin{array}{l}\text { FEM } \\
\text { Aas/ }\end{array}$ \\
\hline ACT. INDEFINIDO \{un\} & /un/ & lunal & & lunos/ & funas/ \\
\hline ACT UNIVERSAL (nil) : & lel/ & $\mathrm{ha}$ & /lo/ & $10 /$ & $10 \%$ \\
\hline & $10 /$ & 101 & & & \\
\hline & lun/ & luna/ & & & \\
\hline
\end{tabular}


En quechua la configuración morfológica está directamente relacionada con el tipo de función sintáctica que cumple la frase nominal. Esto ocurre en especial para la función de actualización definida. Se observa que en la FN sujeto el sufijo -qa se encuentra en oposición interna con los otros morfos que toma prestados la actualización definida (el pluralizador, el genitivo y los situadores posesivos y demostrativos). Con los situadores, el mecanismo de manifestación es el de inclusión semántica. En la FN complemento, la oposición interna está dada entre los morfos de caso y los situadores. Con el morfo de caso, el mecanismo de manifestación es de un amalgamiento de valores: el morfo manifiesta no sólo el caso que le es propio, sino simultáneamente la función de actualizador definido.

Cuadro $\mathrm{N}^{\mathrm{D}} 4$

CONFIGURACION MORFOLOGICA DE LA ACTUALIZACIÓN EN QUECHUA

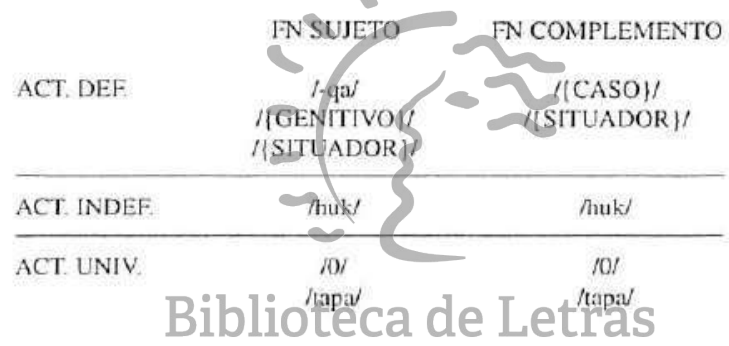

La oposición semantiea entrei las liescerases de vactualización se da de manera absoluta en cada lengua.

EQUIVALENCIAS FORMALES

\title{
I. ACTUALIZACION DEFINIDA
}

\author{
1. Cast. $\{$ EL $\}$ en $/$ FN_Suj./ = Qech. $/$-qa/ en $/$ FN_Suj./ \\ $/\{$ genitivo $\} /$ \\ /\{pluralizador\}/ \\ /\{situador posesivo\}/ \\ /\{situador localizador $\} /$
}


Esta equivalencia formal expresa que en castellano el morfema de la actualización definida (artículo definido), ubicado en el ámbito de una Frase Nominal en función de sujeto, tiene por equivalentes formales hasta cinco formas de manifestación de la actualización definida en quechua. Estos son en orden de importancia: el \{situador localizador\} (adjetivos demostrativos), el \{situador posesivo\} (sufijos de persona poseedora) y el sufijo de actualización definida /-qa/. Los dos primeros presentan entre sí una oposición relativa; pero ambos, en grupo, se oponen de modo absoluto al actualizador/-qa/. Otros dos elementos que, también, manifiestan esta actualización son el caso caso genitivo /-pa/ y el empleo del pluralizador/-kuna/. El primero, porque expresa la relación de posesión y por lo tanto, presenta un elemento definido; y el segundo, debido a que hace necesario el uso del actualizador indefinido para adquirir esta condición. vg.

Huk warmikunas hamuranku, '(Dicen que) unas mujeres vinieron' frente a Warmikunas hamuranku, '(Dicen que) las mujeres vinieron'. La presencia simultánea de cualquiera de estos elementos conel sufijo /-qa/ anula la función actualizadora de éste, que deviene en topicalizador o enfático.

Ejemplos:

1. a) El muchacho se cayó.

b) La mujer se cayó.

Warma-qa urmakurqa.

Warmi-qa urmakurqa.

c) Los hombres sechberōoteca dQ Qarikutămakurqan.

d) Las mujeres se sfyerenPuccinelliWarmikinzourmakurqan.

2. La puerta de la casa se abrió.

3. La puerta de mi casa se abrió.

4. El nieto de Rosa se cayó.

5. El muchacho ése se cayó.

6. La puerta mía se abrió.
Wasi-pa punku-n kichakurqa.

Wasi-y-pa punku-n kichakurqa.

Rosa-pa wilka-n urmakurqa.

Wak warma-qa urmakurqa.

Punku-y-qa kichakurqa.

En el ejemplo 1, se observa una relación directa de equivalencia entre el morfema $\{E L\}$ del castellano, en sus distintas formas flexionadas de expresión, y el morfo /-qa/ del quechua. el morfo de manifestación principal del actualizador quechua \{NIL\}. Los ejemplos 2,3 y 4 presentan la equivalencia entre el morfema \{EL\} del castellano y la manifestación del caso /\{geni tivo $/^{13}$, que simultáneamente a su función principal, cumple la función de

La configuración morfológica del \{genitivo \} en quechua se realiza conjuncionando el sufijo de caso /-pa/ y los sufijos posesivos: Rosa-pa-wilka-n 'el nieto de Rosa' 
actualizador definido. Los ejemplos 5 y 6 muestran la oposición total de los posesivos y los demostrativos frente al morfo principal de manifestación de la actualización definida /-qa/; que como puede observarse queda neutralizado en su función actualizadora y pone de manifiesta su función enfática. En castellano, al parecer sucede el mismo fenómeno; pues el empleo de los artículos definidos en el mismo contexto sintáctico con demostrativos (5) y posesivos (6), nuetraliza su función actualizadora y pone de relieve su función de enfatizador.

2. Cast. $\{$ EL $\}$ en $/$ FN_Compl. $=$ Qech. $\quad\{$ caso $\}$

en /FN_Compl./

\{sit. posesivo\}

\{sit. localizador\}

En el ámbito de la FN en función de complemento, el morfema de la actualización definida del castellano (artículo definido), es el equivalente formal de tres formas de manifestación de la actualización definida en quechua. Estos son los sufijos de persona poseedora, los adjetivos demostrativos y los sufijos de caso. No existe ninguna oposición absoluta entre estos tres elementos, pues la función actualizadora la realizan concomitantemente a su función primordial; y por ello, no es notorioel fenómeno de la neutralización de la función actualizadora. La presencia del sufijo/qa/ en este ámbito, únicamente puede darse en su función topicalizadora o enfática por la presencia obligatoria de los sufijos de $\{$ caso $\}$.

1. Saturnino compró ła quena Satuku gina-ta rantirga,

2. Saturnino come en jel mercado. Satukư plaza-pi mikun.

3. Saturnino compró la quena para el chico. Satuku warma-paq qina-ta rantirqa.

4. Saturnino es el padre del chico. Satuku warma-pa tayta-n-mi.

5. Cierra la puerta! Wiqchay chay punku-ta.

6. Saturnino va a su casa. Satuku wasi-n-man rin.

7. Saturnino se lava las manos. Satuku maki-n-ta mayllakun.

Los ejemplos 1, 2 y 3 muestran, en quechua, el empleo de los sufijos de caso acusativo /-ta/, locativo/-pi/, y benefactivo/-paq/ como correspondencias formales del actualizador $\{E L\}$ del castellano. 4 presenta en quechua, el genitivo /-pa/ y el sufijo posesivo de $3 \mathrm{ra}$. persona / $\mathrm{n} /$ como los equivalentes formales del actualizador definido $\{E L\}$ del castellano. 5 evidencia una cercanía mayor entre el situador demostrativo del quechua y el actualizador \{EL\} 
del castellano. 6 muestra la correspondencia entre los sufijos posesivos del quechua con los posesivos del castellano. 7 muestra una situación especial; en quechua, el sufijo de posesión y el sufijo de caso acusativo asumen la función actualizadora concomitantemente a sus funciones principales; en castellano, se prefiere mantener el actualizador definido, siempre y cuando el contexto lingüístico y/o situacional aclaren quién es el poseedor.

Como se observa la actualización definida en quechua tiene un complejo sistema de manifestación; sin embargo, es evidente el carácter económico del mismo, al evitar la reiteración constante del artículo definido como ocurre en el castellano.

\section{ACTUALIZACION INDEFINIDA \\ Cast. $\{\mathrm{UN}\}$ en $/ \mathrm{FN} /=$ Qech. $/ \mathrm{huk} / \mathrm{en} / \mathrm{FN} /$}

El actualizador indefinido del castéllano $\{$ UN $\}$ (artículo indefinido) se encuentra en equivalencia formal con el actualizador indefinido del quechua / huk/, en el ámbito de una FN en cualquier función. El actualizador indefinido del quechua exige una limitacion sintagmático o contexto lingüístico: la ausencia de los situadores posesivos. La razón es, que se encuentra en oposición absoluta con los sufijosposesiyos, dos adjetivos demostrativos y el sufijo /-qa/ , por ser manifestadores de la actualizacion definida. La presencia de los posesivos neutralizan su funciónactulicidora, a la inversa, su presencia conjunto con un sufijo /qa/ neutraliza la función actualizadora de éste y relieva su función de focalizador o tópico; y con el situador localizador su coexistencia en la misma FN resulta agramatical. Su presencia conjunta con los sufijos de caso produce la neutralización de la función actualizadora de éstos últimos, lo cual posibilita su función de actualizador indefinido en la FN en función de complemento.

1. Un muchacho compró la quena.

Huk warma qinata rantirqa.

2. Uno de tus hijos vino hoy.

Huk wawa-yki kunan hamurqa.

3. Un muchacho, compró la quena.

Huk warma-qa qinata rantirqa.

4. Ese un muchacho compró la quena.*

Chay huk warma qinata rantirqa.*

5. Un muchacho compró una quena.

Huk warma huk qina-ta rantirqa.

6. El muchacho va a una casa.

Warmaqa huk wasi-man rin. 
El ejemplo 1 muestra a $\{\mathrm{UN}\}$ del castellano y $\{$ huk $\}$ del quechua como equivalentes formales por cumplir la función de actualizador indefinido en la FN sujeto. El ejemplo 2 presenta en quechua, la neutralización de la función actualizadora de $/ \mathrm{HUK} /$ por efecto de la copresencia del sufijo de posesión en la misma $\mathrm{FN}^{14}$. En 3 se observa la capacidad de neutralización de /HUK/ frente a /qa/; convirtiéndolo en focalizador. En 4 se verifica la agramaticalidad tanto en quechua como en castellano de la concurrencia del actualizador indefinido con el situador localizador. 5 y 6 presentan la capacidad de neutralización del actualizador indefinido del quechua /HUK/ frente a los sufijos de caso /-ta/ y / -man/, y en general, de cualquier otro.

\section{ACTUALIZACION UNIVERSAL}

1. Cast. /EL/ en /FN_Suj./ = Qech. $10 /$ en /FN_Suj./

El actualizador universal en castellano es un morfema nil que presenta como morfo de expresión a /EL L (artículo definido), en el ámbito de la FN sujeto. Su equivalente formal en quechua es el morfo cero $/ 0 /$, o el indefinido /Tapa/. Ejemplos: Biblioteca de Letras

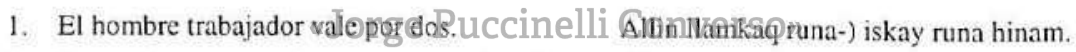

2. Las mujeres campesinas son muy trabajadoras. Chacra warmikuna- 0 allin Ilamkaq kakun.

3. Los niños son hermosos. Warmakuna-0 sumaqlla kanku.

Todos los niños son hermosos. Llapa warmakuna-0 sumqlla kanku.

Los ejemplos 1 y 2 muestran las equivalencias formales entre las formas /EL/ y /-0/ del quechua y el castellano respectivamente. El ejemplo 3 presenta en castellano dos maneras de expresar la universalidad de la referencia, la primera si el contexto lo permite; y la segunda, con el cuantificador absoluto asegura en el contexto lingüístico esta referencia universal. En quechua, parece ser suficiente el empleo del morfo /0/ para evidenciar esta universalidad; el uso del cuantificador /lapa/ agrega un valor enfático a la expresión.

\footnotetext{
H. En este caso equivale a un adjetivo numeral. Un fenómeno similar ocurre en el castellano donde en la frase nominal un hijo tuvo. un funciona como un adjetivo numeral tal como dos en la frase dos hijos niyos.
} 
2. Cast. /0/ en /FN_Compl./= Qech. /El/ +sing. /un/

En el ámbito de la FN complemento, el castellano presenta como forma principal de expresión el morfo /o/, secundariamente el artículo definido con la limitación sintagmático de FN en singular. Tiene también como correspondiente formal en el quechua, a un morfo $/ 0 /$ o al indefinido /tapa/. En quechua, la función de complemento que se ha estudiado es la de predicativo. Ejemplos:

1. Los indios son 0 hombres trabajadores.

2. Las mujeres campesinas son 0 muy trabajadoras.

3. Los niños pequeños son todos (niños) hermosos.

4. Todos los niños pequeños son 0 (niños) hermosos.

Qechua runa-0 allin llamkaq runa-0 kanku. Chacra warmikuna-0 allin llamkaq-0 kakun. Warmachakuna-0 lliw sumaqlla-0 kanku.

Llapa warmachakuna-0 sumaqlla-0 kanku.

Se observan en estos 3 ejemplos que existe una suerte de concordancia de actualización universal entre el sujeto y el complemento predicativo, tanto en el quechua como en el castellano, y que por lo tanto no sólo afectaría a la FN de sujeto o de complemento predicativo; sino a la oración en su conjunto. El ejemplo 3 muestra para el quechua que el cuántificador universal tiene valor enfático tanto en su uso en la FN sujeto como en la de FN comp. predicativo. En castellano, este valor enfático del cuantificador/todos/ se da en la FN complemento predicativo.

\section{Conclusiones}

\section{Biblioteca de Letras}

1. La conclusión más importante es la existencia de una función de actualización nominal que presenta tres tipos básicos: definida, indefinida y universal y que es posible percibir su presencia en el castellano y el quechua, dos lenguas tipológicamente divergentes.

2. Las semejanzas funcionales en el nivel más general del plano del contenido son las que permiten los puntos de encuentro estructural entre ambas lenguas, expresadas en los equivalentes formales.

3. Los equivalentes formales de la actualización manifiestan una función similar mediante limitaciones sintagmáticas precisas para su realización en cada lengua. Por lo tanto, los morfemas y los morfos de expresión establecidos para cada lengua constituyen equivalentes formales entre sí y tentativamente pueden ser asumidos como transferencias positivas en el proceso de aprendizaje de una segunda lengua. 
4. Metodológicamente se puede plantear la necesidad de evaluar no sólo los planteamientos teóricos actuales, sino también evaluar y revalorar planteamientos de otras corrientes y de periodos anteriores. Esto nos permitirá tentar nuevos caminos o hacer nuevos progresos dentro de una senda teórica ya establecida.

5. Es necesario relativizar el concepto de la monovalencia de los morfemas gramaticales como el fenómeno más general. En realidad, la polisemia y la homonimia, fenómenos semánticos muy aceptados para los morfemas lexicales parecen estar presentes en un buen número de casos.

\section{BIBLIOGRAFIA}

BELLO, Andrés y J. Cuervo

1891 Gramática de la lengua castellana. Ed. A. Roger y F.

CATTFORD, J. C.

Chernoviz. París.

$1967 \quad$ A Linguistic Theory of Translation.

COSERIU, Eugene

1978 Gramáticq, semántica y universales. Ed. Gredos. Madrid.

1973 Teoraldel lenguafe y linguistied gentral. Ed. Gredos.

LYONS, John Madridorge Puccinelli Converso»

1971

MENESES, Norma

Introducción en la Lingiiística Teórica. Ed. Teide. Madrid.

1995

La actualización nominal en castellano y quechua. Tesis de licenciatura en Lingüística. Facultad de Letras. UNMSM.

STOCKWELL, Robert, J. Donald Bowen, John W. Martin

1965

The grammatical structures of English and Spanish. WEINRICH, Uriel

University of Chicago Press. Chicago.

1974

Lenguas en contacto. Universidad Central de Venezuela. Caracas.

ZUÑIGA, Madeleine

1974

La Educación Bilingüe y la enseñanza de la pronunciación castellana a los niños quechuahablantes. UNMSM. CILA. Doc. $\mathrm{N}^{\circ} 29$. Lima. 